\title{
Letter to the editor: “COVID-19 does not reduce stroke-related emergencies"
}

\author{
Stefano Caproni ${ }^{1} \cdot$ Chiara Di Schino $^{1} \cdot$ Franco Costantini $^{1} \cdot$ Giorgio Parisi $^{2} \cdot$ Carlo Colosimo $^{1}$ (D)
}

Received: 15 May 2020 / Accepted: 9 July 2020 / Published online: 17 July 2020

(c) Belgian Neurological Society 2020

Keywords COVID-19 $\cdot$ Emergency neurology $\cdot$ Stroke

\section{Dear Editor,}

On the 8th March 2020, the Italian Government ordered a nationwide travel ban, quarantine and lockdown [1] to limit the spread of severe acute respiratory syndrome coronavirus 2 (SARS-CoV-2)-induced disease (COVID-19) [2]. The burden of COVID-19 epidemic has been very severe, with more than 230,000 cases and over 30,000 deaths among Italian residents by the end of May [3]. The national and regional health systems in Italy have attempted to tackle the outbreak by suspending routine outpatient clinics, stopping elective procedures and providing treatment only for highly selective acute cases in order to satisfy the rapidly increasing need for intensive-care beds for COVID-19 patients, most of which are in dedicated, largely reconverted hospitals that are distinct from "COVID-free hospitals".

These measures have had consequences on well-established emergency networks, including the stroke network, which in Italy is prevalently organized according to the hub and spoke system. Indeed, colleagues from Lombardy (the Italian region affected most during the COVID-19 epidemic) have observed a considerable decrease in the number of patients with a stroke requiring acute treatment [4]. The two main reasons that have been hypothesized for this surprising finding are the reluctance of patients with a mild neurological deficit to go to hospital during the COVID-19 outbreak and the failure to recognize a concurrent stroke in patients with respiratory COVID-19 symptoms. Another factor is that it has not been easy for patients to be seen by their

Stefano Caproni

s.caproni@aospterni.it

1 Department of Neurology, "Santa Maria" University Hospital, via Tristano di Joannuccio, 05100 Terni, Italy

2 Emergency Department, "Santa Maria” University Hospital, via Tristano di Joannuccio, 05100 Terni, Italy general practitioners. In light of this, an optimized cooperation between COVID-19-ward-physicians and neurologists is warranted because underdiagnosis of stroke, Guillain-Barré syndrome and other COVID-related neurological disorders negatively influences outcome.

The data we present here suggest that there has not been a reduction in the number of patients presenting with severe neurological emergencies at hospitals during the COVID-19 outbreak. The Department of Neurology at the Santa Maria University Hospital in Terni, in which we work, serves as a comprehensive stroke center for southern Umbria. We collected data on the neurological consultations and admissions from the Emergency Department between 08:00 AM on 26th March and 02:00 PM on 27th March 2020, and we compared these results with similar periods ( 2 working days) in November 2018 and 2019, to determine whether there was any reduction in acute neurological events (Table 1). Furthermore, of the 44 patients admitted to the neurology ward during the first month of lockdown, 11 underwent acute stroke treatment (4 intravenous thrombolysis, 1 mechanical thrombectomy and 6 both treatments). These data are similar to those from the previous 2 years.

Although there has been an overall reduction in the total number of admissions to the Emergency Department during the COVID-19 outbreak in the Umbria region, our data indicate that the percentage of patients requiring an acute neurological consultation remained stable (11.1\% vs. $10 \%$ in 2018 and $12.6 \%$ in 2019). Furthermore, despite the fall in the overall number of hospital admissions (16.7\% vs $22.3 \%$ in 2018 and $30.5 \%$ in 2019), admissions to the neurology ward increased if compared with the previous 2 years $(50 \%$ vs. $7.7 \%$ in 2018 and $9.5 \%$ in 2019). These outcomes may be explained by a drop in the number of requests for neurological consultations in general whereas the number of admissions to the neurology ward confirms that serious neurological events requiring hospitalization did not decrease 
Table 1 Emergency department admissions and neurological consultations during the COVID-19 outbreak and in similar periods in 2018 and 2019

\begin{tabular}{llllll}
\hline Year & Patients & Total & Discharged & Admitted & $\begin{array}{c}\text { Percentage } \\
\text { of admitted }\end{array}$ \\
\hline 2018 & Emergency department & 130 & 101 & 29 & 22.3 \\
& Neurological consultations & 13 & 12 & 1 & 7.7 \\
& Neurological consultations/total patients *100 & 10 & 11.9 & 3.4 & \\
2019 & Emergency department & 167 & 116 & 51 & 30.5 \\
& Neurological consultations & 21 & 12 & 2 & 9.5 \\
& Neurological consultations/Total patients *100 & 12.6 & 10.3 & 3.9 & \\
& Emergency department & 54 & 45 & 9 & 16.7 \\
& Neurological consultations & 6 & 3 & 3 & 50 \\
& Neurological consultations/Total patients *100 & 11.1 & 6.7 & 33.3 & \\
\hline
\end{tabular}

in the period considered, which is in line with the expected number of acute treatments performed for ischemic stroke.

According to these data, the COVID-19 epidemic has not significantly altered the incidence of strokes or the prompt admission to a comprehensive stroke center in our central Italian region. A possible explanation for the discrepancy between our data and those reported by other Italian centers may be the significantly lower prevalence of COVID-19 infection in our region than in other regions, particularly those in northern Italy. The prevalence of COVID-19-positive subjects in the resident population has varied considerably in Italy, with figures ranging from 860 per 100,000 in Valle d'Aosta to 50 per 100,000 in Calabria (150 per 100,000 in Umbria) (see https://www.gimbe.org/download/ CS_GIMBE_COVID-19_mappa_rischio_regioni\%20(figur e).pdf). This variability has also been reflected by the temporary relocation/dismissal of neurological units, including stroke units, in some of the regions affected most (to increase the number of beds available for COVID-19 patients).

Whatever the explanation for these discrepant findings in Italy, we would like to stress the need for the establishment of stroke networks and care systems able to deliver highquality emergency stroke care at any given time, including times of crisis, particularly when and where the number of comprehensive stroke centers needs to be reduced for any reason. It thus becomes even more important to increase awareness among health professionals and the members of the public, especially those who are at high risk, of how to recognize a stroke and help launch the network to avoid significant delays in management [5]. Stroke networks should, if necessary, be adjusted to minimize the risk of infection for health professionals and patients when suspected or confirmed COVID-19 patients with stroke are treated. Since COVID-19 patients are affected by enhanced coagulopathy, which is accompanied by a higher risk of arterial and venous thromboembolism that may affect multiple sites (heart, brain, kidney, lung), we expect to observe an increased number of COVID-19-specific or COVID-19-mediated strokes in the coming months. We should, therefore, be prepared to deal with the consequences of any COVID-19 outbreak on neurological care that may result from the increased prevalence of patients with this virus according to specific protocols and the best scientific evidence.

\section{Compliance with ethical standards}

Conflict of interest The authors have nothing to disclose regarding the present study.

Ethical approval All procedures performed in the study involving human participants were in accordance with the ethical standards of the Trust and with the 1964 Helsinki declaration and its later amendments or comparable ethical standards.

Informed consent For this type of study formal consent is not required.

\section{References}

1. Saglietto A, D’Ascenzo F, Zoccai GB, De Ferrari GM (2020) COVID-19 in Europe: the Italian lesson. Lancet 395:1110-1111

2. Wang D, Hu B, Hu C et al (2020) Clinical characteristics of 138 hospitalized patients with 2019 novel coronavirus infected pneumonia in Wuhan, China. JAMA 323:1061-1069

3. Coronavirus COVID-19 Global Cases by the Center for Systems Science and Engineering (CSSE) at Johns Hopkins University (JHU) in https://coronavirus.jhu.edu/map.html [online, accessed 15th May 2020].

4. Bersano A, Pantoni L (2020) On being a neurologist in Italy at the time of the COVID-19 outbreak. Neurology 94(21):905-906

5. Zhao J, Rudd A, Liu R (2020) Challenges and potential solutions of stroke care during the Coronavirus Disease 2019 (COVID-19) Outbreak. Stroke 51(5):1356-1357

Publisher's Note Springer Nature remains neutral with regard to jurisdictional claims in published maps and institutional affiliations. 